\title{
Arithmetic properties of plane partitions
}

\author{
To Doron: a wonderful Mensch
}

\author{
Tewodros Amdeberhan \\ Department of Mathematics \\ Tulane University, New Orleans, LA 70118 \\ tamdeber@tulane.edu \\ Victor H. Moll \\ Department of Mathematics \\ Tulane University, New Orleans, LA 70118 \\ vhm@math.tulane.edu \\ Submitted: Aug 31, 2010; Accepted: Oct 14, 2010; Published: Jan 2, 2011 \\ Mathematics Subject Classification: 05A15, 11B75
}

\begin{abstract}
The 2-adic valuations of sequences counting the number of alternating sign matrices of size $n$ and the number of totally symmetric plane partitions are shown to be related in a simple manner.
\end{abstract}

Keywords: valuations, alternating sign matrices, totally symmetric plane partitions.

\section{Introduction}

A plane partition (PP) is an array $\pi=\left(\pi_{i j}\right)_{i, j \geq 1}$ of nonnegative integers such that $\pi$ has finite support and is weakly decreasing in rows and columns. These partitions are often represented by solid Young diagrams in 3-dimensions. MacMahon found a complicated formula for the enumeration of all PPs inside an $n$-cube. This was later simplified to

$$
\mathrm{PP}_{n}=\prod_{i, j, k=1}^{n} \frac{i+j+k-1}{i+j+k-2}
$$

A plane partition is called symmetric (SPP) if $\pi_{i j}=\pi_{j i}$ for all indices $i, j$. The number of such partitions whose solid Young diagrams fit inside an $n$-cube is given by

$$
\operatorname{SPP}_{n}=\prod_{j=1}^{n} \prod_{i=1}^{n} \frac{i+j+n-1}{i+j+i-2}=\prod_{j=1}^{n} \prod_{i=j}^{n} \frac{i+j+n-1}{i+j-1}
$$

Another interesting subclass of partitions is that of totally symmetric plane partitions (TSPP). These are symmetric partitions $\pi$ such that every row of $\pi$ is self-conjugate as 
an ordinary plane partition (or the Young diagrams are invariant under any permutation of the axes). J. Stembridge [3] showed that the number of TSPP in an $n$-cube is given by

$$
\operatorname{TSPP}_{n}=\prod_{1 \leq i \leq j \leq k \leq n} \frac{i+j+k-1}{i+j+k-2}=\prod_{j=1}^{n} \prod_{i=j}^{n} \frac{i+j+n-1}{i+j+i-2}=\prod_{1 \leq i \leq j \leq n} \frac{i+j+n-1}{i+j+j-2} .
$$

For the solid Young diagram of a plane partition $\pi$ that fits inside a box of a given size, one can take the collection of cubes that are in the box but do not belong to the solid Young diagram. These determine another plane partition called the complement of $\pi$. If the complement of $\pi$ is the same as the original partition, $\pi$ is called self-complementary. Such partitions only fit in an even-dimensional box. The number of plane partitions inside a $2 n \times 2 n \times 2 n$ box that are both totally symmetric and self-complementary (TSSCPP) is given by

$$
\operatorname{TSSCPP}_{2 n}=\prod_{1 \leq i \leq j \leq n} \frac{i+j+n-1}{i+j+i-1} .
$$

The proof required the efforts of three combinatorialists: W. F. Doran, J. Stembridge and G. Andrews.

An alternating sign matrix (ASM) is an array of 0,1 and -1 such that the entries of each row and column add up to 1 and the non-zero entries of a given row/column alternate. After a fascinating sequence of events, D. Zeilberger [5] completely proved the conjecture that the number of ASM of size $n$ equals $\operatorname{TSSCPP}_{2 n}$. Bressoud's book [1] contains an entertaining story of these counting functions.

Note. For simplicity, we write $A_{n}=\operatorname{TSSCPP}_{2 n}, B_{n}=\mathrm{TSPP}_{n}$ and $T_{n}=\mathrm{PP}_{n}$.

A simple calculation shows that $A_{n}$ and $B_{n}$ do not divide each other as integers. The first few values of the quotient $A_{n} / B_{n}$ are given by

$$
\frac{1}{2}, \frac{2}{5}, \frac{7}{16}, \frac{7}{11}, \frac{39}{32}, \frac{52}{17}, \frac{3211}{320}, \frac{988}{23}, \frac{30685}{128}, \frac{50540}{29} .
$$

The quotient $A_{n} / B_{n}$ presents a large amount of cancellation. For instance, the integers $A_{40}, B_{40}$ have 182 and 100 digits and the reduced form of $A_{n} / B_{n}$ has denominator 17. Motivated by this cancellation, during a conference in the summer of 2010 at Nankai University, where Manuel Kauers explained the remarkable result [2], one of the authors computed a list of the values when $B_{n}$ is odd. This question had also been the key to the main ideas behind the arithmetic properties of $A_{n}$, as described in [4]. Figure 1 depicts the 2 -adic valuation of $A_{n}$.

The computation showed that the indices where $B_{2 n}$ is odd is related to the Jacobsthal numbers that are defined by the recurrence $J_{n}=J_{n-1}+2 J_{n-2}, J_{0}=1$ and $J_{1}=1$. These are precisely the indices where $A_{n}$ is odd. This observation lead to the first result in this paper.

Note. For $n \in \mathbb{N}$, denote by $\nu_{2}(n)$ the 2-adic valuation of $n$, defined as the highest power of 2 that divides $n$. Let $s_{2}(n)$ equal to the sum of the binary digits of $n$. 


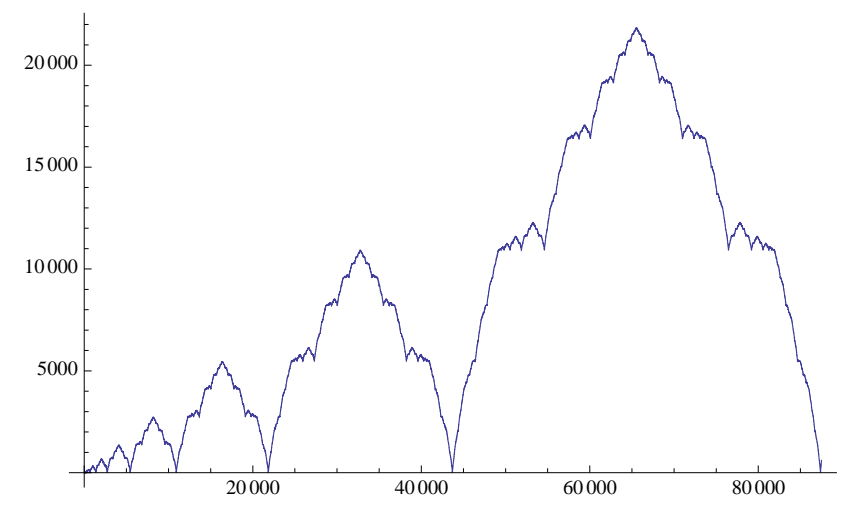

Figure 1: The 2-adic valuation of $A_{n}$

Theorem 1.1 For $n \in \mathbb{N}$. Then,

$$
\begin{aligned}
\nu_{2}\left(B_{2 n}\right) & =\nu_{2}\left(A_{n}\right) \\
\nu_{2}\left(B_{2 n-1}\right) & =\nu_{2}\left(A_{n}\right)+2 n-1 .
\end{aligned}
$$

Proof. To compare $A_{n}$ with $B_{2 n}$, compute the ratios

$$
\begin{aligned}
\frac{A_{n+1}}{A_{n}} & =\prod_{j=1}^{n+1} \prod_{i=1}^{j} \frac{i+j+n}{i+j+i-1} \prod_{j=1}^{n} \prod_{i=1}^{j} \frac{i+j+i-1}{i+j+n-1} \\
& =\frac{3 n+2}{n+1} \prod_{i=1}^{n}(i+2 n+1)(i+2 n) \prod_{j=1}^{n-1} \frac{1}{2 j+n+1} \prod_{i=1}^{n+1} \frac{1}{i+i+n} \\
& =\prod_{i=1}^{n} \frac{(i+2 n+1)(i+2 n)}{(2 i+n-1)(2 i+n)}
\end{aligned}
$$

and

$$
\begin{aligned}
\frac{B_{2 n+2}}{B_{2 n}} & =\prod_{k=1}^{2 n+2} \prod_{j=1}^{k} \prod_{i=1}^{j} \frac{i+j+k-1}{i+j+k-2} \prod_{k=1}^{2 n} \prod_{j=1}^{k} \prod_{i=1}^{j} \frac{i+j+k-2}{i+j+k-1} \\
& =\prod_{j=1}^{2 n+1} \prod_{i=1}^{j} \frac{i+j+2 n}{i+j+2 n-1} \prod_{j=1}^{2 n+2} \prod_{i=1}^{j} \frac{i+j+2 n+1}{i+j+2 n} \\
& =\frac{(6 n+1)(6 n+3)(6 n+5)}{(2 n+1)(2 n+2)(2 n+3)} \prod_{i=1}^{2 n-1} \frac{(i+4 n+1)(i+4 n+3)}{(2 i+2 n+2)(2 i+2 n+3)} \\
& =\frac{(6 n+5)}{(2 n+1)} \prod_{i=1}^{2 n} \frac{(i+4 n+1)(i+4 n+3)}{(2 i+2 n)(2 i+2 n+1)} \\
& =\frac{(6 n+5)}{(2 n+1)} \frac{\prod_{i=1}^{n}(2 i+4 n+1)(2 i+4 n+3)}{\prod_{i=1}^{2 n}(2 i+2 n+1)} \frac{\prod_{i=1}^{n}(2 i+4 n)(2 i+4 n+2)}{\prod_{i=1}^{2 n}(2 i+2 n)}
\end{aligned}
$$




$$
\begin{aligned}
& =\frac{(6 n+5)}{(2 n+1)} \prod_{i=1}^{n} \frac{(2 i+4 n+1)(2 i+4 n+3)}{(4 i+2 n+1)(4 i+2 n-1)} \prod_{i=1}^{n} \frac{(2 i+4 n)(2 i+4 n+2)}{(4 i+2 n)(4 i+2 n-2)} \\
& =\frac{(6 n+5)}{(2 n+1)} \prod_{i=1}^{n} \frac{(2 i+4 n+1)(2 i+4 n+3)}{(4 i+2 n+1)(4 i+2 n-1)} \prod_{i=1}^{n} \frac{(i+2 n)(i+2 n+1)}{(2 i+n)(2 i+n-1)} \\
& =\frac{(6 n+5)}{(2 n+1)} \prod_{i=1}^{n} \frac{(2 i+4 n+1)(2 i+4 n+3)}{(4 i+2 n+1)(4 i+2 n-1)} \times \frac{A_{n+1}}{A_{n}} .
\end{aligned}
$$

Since $\nu_{2}\left(B_{2}\right)=\nu_{2}\left(A_{1}\right)=0$ and $\nu_{2}\left(B_{2 n+2}\right)-\nu_{2}\left(B_{2 n}\right)=\nu_{2}\left(A_{n+1}\right)-\nu_{2}\left(A_{n}\right)$, the first assertion follows. Similarly,

$$
\begin{aligned}
\frac{B_{2 n+1}}{B_{2 n}} & =\prod_{i=1}^{n+1} \frac{(2 i+4 n+1)(2 i+2 n)}{(4 i+2 n-1)(4 i+2 n-3)} \times \frac{A_{n+1}}{A_{n}} \\
& =2^{n+1} \frac{(2 n+1) !}{n !} \prod_{i=1}^{n+1} \frac{(2 i+4 n+1)}{(4 i+2 n-1)(4 i+2 n-3)} \times \frac{A_{n+1}}{A_{n}} .
\end{aligned}
$$

Hence

$$
\nu_{2}\left(B_{2 n+1}\right)-\nu_{2}\left(B_{2 n}\right)=n+1+2 n+1-s_{2}(2 n+1)-n+s_{2}(n)+\nu_{2}\left(A_{n+1}\right)-\nu_{2}\left(A_{n}\right),
$$

where Legendre's formula $\nu_{2}(m !)=m-s_{2}(m)$ is applied. The rest follows from $s_{2}(2 n+$ $1)=s_{2}(n)+1$ and the first part of the proof.

\section{A product identity}

In this section we consider the function $\mathrm{SPP}_{n}$ counting the number of symmetric plane partitions of size $n$. Recall

$$
\operatorname{SPP}_{n}=\prod_{j=1}^{n} \prod_{i=1}^{n} \frac{i+j+n-1}{i+j+i-2}
$$

The next result appears to be new and is similar to

$$
\text { cylindrically symmetric }=(\text { totally symmetric })^{2} \text {. }
$$

Theorem 2.1 The identity $S P P_{n}=\operatorname{TSSCPP}_{2 n} \times \operatorname{TSPP}_{n}$ holds.

Proof: After some regrouping and re-indexing,

$$
\begin{aligned}
\operatorname{TSSCPP}_{2 n} & =\prod_{j=1}^{n} \prod_{i=1}^{j} \frac{i+j+n-1}{i+j+i-1} \\
& =\prod_{j=1}^{n} \prod_{i=1}^{j}(i+j+n-1) \prod_{j=2}^{n} \prod_{i=1}^{j-1}(i+j+i-2)^{-1} \prod_{i=1}^{n}(2 i+n-1)^{-1},
\end{aligned}
$$


and

$$
\begin{aligned}
\operatorname{TSPP}_{n} & =\prod_{j=1}^{n} \prod_{i=j}^{n} \frac{i+j+n-1}{i+j+i-2} \\
& =\prod_{j=1}^{n} \prod_{i=j+1}^{n}(i+j+n-1) \prod_{j=2}^{n} \prod_{i=j}^{n}(i+j+i-2)^{-1} \prod_{j=1}^{n}(2 j+n-1) \prod_{i=1}^{n}(2 i-1)^{-1} .
\end{aligned}
$$

Combining the two it follows that

$$
\begin{aligned}
\operatorname{TSSCPP}_{2 n} \times \operatorname{TSPP}_{n} & =\prod_{j=1}^{n} \prod_{i=1}^{n}(i+j+n-1) \prod_{j=2}^{n} \prod_{i=1}^{n}(i+j+i-2)^{-1} \prod_{i=1}^{n}(2 i-1)^{-1} \\
& =\prod_{j=1}^{n} \prod_{i=1}^{n}(i+j+n-1) \prod_{j=1}^{n} \prod_{i=1}^{n}(i+j+i-2)^{-1} \\
& =\prod_{j=1}^{n} \prod_{i=1}^{n} \frac{i+j+n-1}{i+j+i-2} \\
& =\operatorname{SPP}_{n} .
\end{aligned}
$$

The next statement follows from Theorem 1.1 and Theorem 2.1.

Corollary 2.2 For $n \in \mathbb{N}$,

$$
\nu_{2}\left(S P P_{2 n}\right)=\nu_{2}\left(A_{2 n}\right)+\nu_{2}\left(A_{n}\right)
$$

and

$$
\nu_{2}\left(S P P_{2 n-1}\right)=\nu_{2}\left(A_{2 n-1}\right)+\nu_{2}\left(A_{n}\right)+2 n-1 .
$$

\section{$3 \quad$ Some conjectures}

This last section contains some conjectures. The first one deals with the 2-adic valuation of the sequences $B_{n}$ and $T_{n}$.

Conjecture 3.1 For $n \in \mathbb{N}$, the inequalities

$$
\nu_{2}\left(T_{2 n}\right)>\nu_{2}\left(B_{2 n}\right) \text { and } \nu_{2}\left(T_{2 n+1}\right)<\nu_{2}\left(B_{2 n+1}\right)
$$

hold.

The second conjecture is related to sequences formed by successive ratios.

Given a sequence of positive numbers $\left\{a_{n}\right\}$ consider the successive ratios defined by

$$
a_{n+1}^{\{0\}}:=a_{n+1} \text { and } a_{n+1}^{\{k\}}:=a_{n+1}^{\{k-1\}} / a_{n}^{\{k-1\}} .
$$


For instance,

$$
a_{n+1}^{\{1\}}=\frac{a_{n+1}}{a_{n}} \text { and } a_{n+1}^{\{2\}}=\frac{a_{n+1} a_{n-1}}{a_{n}^{2}} .
$$

In particular $a_{n}$ is nonincreasing if $a_{n+1}^{\{1\}} \leq 1$ and logconcave if $a_{n+1}^{\{2\}} \leq 1$ and logconvex if $a_{n+1}^{\{2\}} \geq 1$.

Conjecture 3.2 Let $A_{n}$ be the ASM sequence. For $0 \leq k \leq 3$ the iterated sequence $A_{n+1}^{\{k\}}$ is logconvex. For $k \geq 4$, the sequence $A_{n+1}^{\{k\}}$ is logconvex when $k$ is odd and logconcave when $k$ is even.

Problem 3.3 Find a combinatorial proof of Theorem 2.1.

Note. The calculations were performed after the talk. There were no violations to the Zeilberger rules of order.

\section{Acknowledgement}

The authors are grateful to G. Andrews, R. Stanley, J. Stembridge and D. Zeilberger for sharing their insight into the questions presented in this paper. The authors also thank the referee for a careful reading of the manuscript. The work of the second author was partially funded by NSF-DMS 0713836 .

\section{References}

[1] D. Bressoud. Proofs and Confirmations: the story of the Alternating Sign Matrix Conjecture. Cambridge University Press, 1999.

[2] C. Koutschan, Manuel Kauers, and D. Zeilberger. A proof of George Andrews's and David Robbins $q$-TSPP Conjecture. Preprint, 2010. http://arxiv.org/abs/1002. 4384

[3] J. Stembridge. The enumeration of totally symmetric plane partitions. Adv. Math., 111:227-243, 1995.

[4] X. Sun and V. Moll. The p-adic valuation of sequences counting Alternating Sign Matrices. Journal of Integer Sequences, 12:1-21, 2009.

[5] D. Zeilberger. Proof of the Alternating Sign Matrix conjecture. Electronic Journal of Combinatorics, 3:1-78, 1996. 